\title{
Development and Validation of a Nomogram for the Estimation of Response to Platinum-Based Neoadjuvant Chemotherapy in Patients with Locally Advanced Cervical Cancer
}

This article was published in the following Dove Press journal:

Cancer Management and Research

\begin{abstract}
Wei Chen'
Bo Wang ${ }^{2}$

Rong Zeng ${ }^{3}$

Tiejun Wang'

'Department of Breast Surgery, Hubei Cancer Hospital, Tongji Medical College, Huazhong University of Science and Technology and Hubei Provincial Clinical Research Center for Breast Cancer, Wuhan, Hubei, 430079, People's Republic of China; ${ }^{2}$ Department of Obstetrics and Gynecology, Tongji Hospital, Tongji Medical College, Huazhong University of Science and Technology, Wuhan, People's Republic of China; ${ }^{3}$ Department of Obstetrics and Gynecology, Hubei Cancer Hospital, Tongji Medical College, Huazhong University of Science and Technology, Wuhan, People's Republic of China
\end{abstract}

Correspondence: Tiejun Wang Department of Breast Surgery, Hubei Cancer Hospital, Tongji Medical College, Huazhong University of Science and Technology and Hubei Provincial Clinical Research Center for Breast Cancer, II6 Zhuodaoquan South Road, Hongshan District, Wuhan, Hubei Province, People's Republic of China

Tel +86-|32-779|-4596

Email tiejunwanghp@I63.com
Purpose: Non-response to platinum-based neoadjuvant chemotherapy (non-rNACT) reduces the surgical outcomes of patients with locally advanced cervical cancer (LACC). The development of an accurate preoperative method to predict a patient's response to NACT (rNACT) could help surgeons to manage therapeutic intervention in a more appropriate manner.

Patients and Methods: We recruited a total of 341 consecutive patients who underwent platinum-based NACT followed by radical surgery (RS) at the Hubei Cancer Hospital between January 1, 2010 and April 1, 2020. All patients had been diagnosed with stage Ib2IIa2 cervical cancer in accordance with the 2009 International Federation of Gynecology and Obstetrics (FIGO) classification system. First, we created a training cohort of patients who underwent NACT + RS $(n=239)$ to develop a nomogram. We then validated the performance of the nomogram in a validation cohort of patients who underwent NACT $+R S(n=102)$. Data analysis was conducted from October 1, 2020. First, we determined overall survival (OS) and progression-free survival (PFS) after NACT+RS. Multivariate logistic regression was then used to identify independent risk factors that were associated with the response to rNACT; these were then incorporated into the nomogram.

Results: The analysis identified several significant differences between the rNACT and nonrNACT groups, including neutrophil-lymphocyte ratio (NLR), platelet lymphocyte ratio (PLR), lymphocyte monocyte ratio (LMR), platelet count, and FIGO stage. The performance of the rNACT nomogram score exhibited a robust $\mathrm{C}$-index of 0.76 (95\% confidence interval [CI]: 0.65 to 0.87 ) in the training cohort and high C-index of 0.71 (95\% CI: 0.62 to 0.78 ) in the validation cohort. Clinical impact curves showed that the nomogram had good predictive ability.

Conclusion: We successfully established an accurate and optimized nomogram that could be used preoperatively to predict rNACT in patients with LACC. This model can be used to evaluate the risk of an individual patient experiencing rNACT and therefore facilitate the choice of treatment.

Keywords: locally advanced cervical cancer, neoadjuvant chemotherapy, clinical response, nomogram prediction, prognosis

\section{Introduction}

Globally, more than half a million women are diagnosed with cervical cancer each year and over 300,000 patients die of this form of malignancy. ${ }^{1}$ Cervical cancer is one of the most common forms of gynecological tumors. However, due to the careful management of surgical treatment and concurrent chemoradiotherapy, the majority of patients with early-stage cervical cancer can achieve good 
levels of recovery, at least to some extent. ${ }^{2}$ However, the survival outcomes of patients with LACC remain low, leading to the widespread application of neoadjuvant chemotherapy (NACT) in an attempt to improve the prognosis of patients with LACC. ${ }^{3,4}$ Previous researchers have proposed platinum-based NACT, followed by a radical hysterectomy, as a potential alternative, particularly for patients diagnosed with stage Ib2 to IIa on the 2009 International Federation of Gynecology and Obstetrics (FIGO) classification system. ${ }^{5}$ NACT, prior to surgery or radiation therapy, has also been explored as an alternative method with which to reduce tumor bulk; this approach appears to enhance the efficacy of subsequent therapy. ${ }^{6}$ However, while impressive clinical response rates to cisplatin-based NACT are known to play a crucial role in prognosis, it is evident that only patients who achieve a complete clinical response will benefit from the combination of NACT and surgical management. In other words, the response to NACT (rNACT) plays a decisive role in the prognosis of patients with LACC. ${ }^{6,7}$

At present, the rNACT is estimated by considering the Response Evaluation Criteria In Solid Tumors (RECISR, version 1.1$)^{8}$ after the completion of NACT. On the basis of this evaluation, patients will then either undergo surgery or alternative forms of treatment. However, situations occur when patients fail to respond to NACT (nonrNACT); this situation delays treatment opportunities and creates a scenario that is very unfavorable for the patients. Therefore, estimating a patient's response to NACT preoperatively is vital if we are to make the appropriate treatment decisions. Accurate estimations of rNACT, as judged preoperatively, could help surgeons to decide whether to administer neoadjuvant chemotherapy or not. Consequently, there is an urgent need to develop an accurate model for predicting the outcome of NACT that can be used to facilitate the management of clinical treatment. In the present study, we established a nomogram to predict rNACT. We then analyzed the predictive performance of this nomogram in a deviation cohort and then verified performance in an internal validation cohort.

\section{Patients and Methods}

\section{Patients}

Between January 1, 2010 and April 1, 2020, we prospectively collated data from consecutive patients who had been diagnosed with LACC at the Hubei Cancer Hospital. All patients had received NACT before surgery and had undergone radical hysterectomy. This study was approved by the Institutional Ethics Committee of the Hubei Cancer Hospital (Reference: LLHBCH2020LW023), in compliance with the Declaration of Helsinki. Written informed consent was obtained from all participants before any treatment. All patients' information was anonymous. The inclusion criteria were as follows: (1) patient had been diagnosed with FIGO stage Ib2-IIa2 cervical cancer; (2) squamous or adenocarcinoma of the cervix had been confirmed by pathological examination prior to treatment; (3) patient had received preoperative contrast-enhanced magnetic resonance imaging (MRI) and routine pelvic examination; and (4) patient had undergone NACT ( 2 to 3 cycles of a platinum-based regimen). We excluded patients who had a history of all other malignancies and any patient who had an incomplete set of medical data. Eligible patients who received NACT, followed by radical surgery between January 1, 2010 and April 1, 2016 were included in the training cohort so that we could establish the nomogram. Patients who underwent the same treatment between May 1, 2016 and April 1, 2020 were used for internal validation.

\section{Blood Sample Collection}

Blood samples (3-5 $\mathrm{mL}$ of whole blood) were collected from each patient on an empty stomach on the morning of the day prior to NACT. The samples were analyzed by the laboratory on the same day, including a routine blood test, platelet test, serological test, and coagulation function test.

\section{Preoperative Examination, NACT, and Surgical Management}

Routine preoperative examinations included pelvic examinations, blood routine tests, liver and renal function tests, serum tumor markers tests, and imaging examinations. The NACT protocol included paclitaxel (the first day: $135-175 \mathrm{mg} / \mathrm{m}^{2}$, continuous intravenous infusion; interval time: 21 days) and carboplatin (the second day: area under the curve [AUC] was equal to 5; continuous intravenous infusion; interval time: 21 days). The surgical approach included radical hysterectomy plus pelvic lymphadenectomy. Patients with pathological risk factors were treated with chemotherapy and/or radiotherapy.

\section{Evaluating the Safety and Efficacy of NACT}

Grade III-IV NACT protocols are associated with "severe toxicity" according to the World Health Organization 
(WHO) grading standards. According to RECIST (version 1.1) criteria, the response to NACT is divided into four levels: (1) complete response (CR) in which the tumor completely disappeared; (2) partial response (PR) in which the diameter of the tumor was reduced by at least $30 \%$; (3) progressive disease (PD) in which the diameter of the tumor was reduced by at least $20 \%$; and (4) stable disease (SD): in which the diameter of the tumor did not shrink sufficiently to qualify for PR but did not increase sufficiently to qualify for PD. ${ }^{9}$

\section{Follow-Up}

After discharge, all patients were monitored regularly in the outpatient clinic. Patients were followed-up every 3 to 6 months in the first 2 years after treatment; every 6 months in the third year, and once annually thereafter. The end points were OS and PFS. OS was measured from the date of $R S$ to the date of the patient's death or the date of the last follow-up visit. PFS was calculated from the date of $R S$ to the date when tumor recurrence was diagnosed.

\section{Statistical Analysis}

Continuous variables are expressed as mean (standard deviation) and compared using the two-tailed $t$ test or the MannWhitney test. Categorical variables were compared using the $\chi^{2}$ test or Fisher's exact test. To explore potential predictive factors, we also calculated the hazard ratio (HR) and the corresponding 95\% confidence interval (CI) from the Cox proportional hazards model. Univariate and multivariate logistic analyses were used to explore the risk factors for rNACT. A nomogram was formulated based on results arising from the multivariate logistic regression analysis. The nomogram was based on the proportional conversion of each regression coefficient in the multivariate logistic regression to a 0 to 100 -point scale. The effect of the variable with the highest $\beta$ coefficient (the absolute value) was assigned 100 points. ${ }^{10}$ Points were added for all independent variables in order to create a total which was then converted to predicted probabilities. Next, we used bootstrapping plots to calculate the concordance index (C-index) and area under the timedependent receiver operating characteristic curve (timedependent AUC) so that we could evaluate our ability to calibrate the curve. Typically, C-index and AUC values that exceeded 0.6 were suggestive of a reasonable estimation. We also used net reclassification index (NRI), integrated discrimination improvement (IDI), and decision curve analysis (DCA), to evaluate the clinical benefits and utility of the nomogram, as described previously. ${ }^{11,12}$ The cut-off point for risk stratifications was selected using X-tile. All analyses were conducted using SAS, version 9.1 (SAS Institute Inc.) and the R statistical package (v.3.6.2; R Foundation for Statistical Computing, Vienna, Austria; https://www.r-pro ject.org). A P-value $<0.05$ was considered to be statistically significant.

\section{Results}

\section{Clinicopathological Characteristics}

During the period of enrollment, 341 consecutive patients with stage Ib2-IIa2 LACC underwent NACT in Hubei Cancer Hospital. Of these, 239 patients met our inclusion criteria and were enrolled in our training cohort. Next, we enrolled our validation cohort; these patients underwent procedures between May 1, 2016 and April 1, 2020 ( $\mathrm{n}=$ 102). Demographics and baseline data are summarized in Table 1. According to the RECIST (version 1.1) criteria, rNACT was identified in $213(89.1 \%)$ and $88(86.3 \%)$ patients in the training and validation cohorts, respectively.

\section{Postoperative Prognosis and Independent Prognostic Factors}

The study was completed on October 1, 2020. The median follow-up time was 60.5 months (range, 14 to 75 months) and 38.7 months (range, 16 to 46 months) for the training and validation cohorts, respectively. In the training cohort, the 1-, 3-, and 5-year OS rates for the patients experiencing rNACT were $95.61 \%, 88.79 \%$, and $83.56 \%$, respectively; the 1-, 3-, and 5-year DFS rates were $91.92 \%$, $86.78 \%$, and $73.21 \%$, respectively. In the validation cohort, the 1-, 3-, and 5-year OS rates of the patient experiencing rNACT were $92.37 \%, 86.19 \%$, and $82.76 \%$, respectively; the 1-, 3-, and 5-year DFS rates were $89.07 \%, 84.65 \%$, and $75.13 \%$, respectively. The results of univariate and multivariate Cox regression analysis are summarized in Table 2. Multivariate analysis showed that rNACT was an independent risk factor for OS and DFS (Figure 1A).

\section{Development and Validation of a Predictive Nomogram for rNACT}

All of the parameters used to construct the nomogram were based on preoperative data, including peripheral blood inflammatory markers (neutrophil-lymphocyte ratio (NLR), platelet lymphocyte ratio (PLR), lymphocyte monocyte ratio (LMR), and platelet count), imaging indicators (eg, the largest tumor diameter), and serum tumor 
Table I Demographics and Baseline Characteristics of the Patients Undergoing NACT

\begin{tabular}{|c|c|c|c|c|}
\hline \multirow[t]{2}{*}{ Variables } & \multirow{2}{*}{$\begin{array}{l}\text { Training Cohort } \\
(n=239)\end{array}$} & \multirow{2}{*}{$\begin{array}{l}\text { Validation Cohort } \\
(n=102)\end{array}$} & \multirow[t]{2}{*}{$\chi / \mathbf{t}$} & \multirow[t]{2}{*}{$P$-value } \\
\hline & & & & \\
\hline Age, years & & & 1.129 & 0.260 \\
\hline $\operatorname{Mean}( \pm S D)$ & $46.4( \pm 25.9)$ & $49.6( \pm 18.6)$ & & \\
\hline Diameter,cm & & & 7.815 & 0.005 \\
\hline$<4$ & 99 (4I.4\%) & $26(25.5 \%)$ & & \\
\hline$\geq 4$ & 140 (58.6\%) & 76 (74.5\%) & & \\
\hline NLR & & & 13.336 & $P<0.01$ \\
\hline$<3.16$ & 146 (61.1\%) & $83(81.4 \%)$ & & \\
\hline$\geq 3.16$ & 93 (38.9\%) & 19 (I8.6\%) & & \\
\hline LMR & & & 70.659 & $P<0.01$ \\
\hline$<3.6$ & 47 (19.7\%) & 68 (66.7\%) & & \\
\hline$\geq 3.6$ & 192 (80.3\%) & 34 (33.3\%) & & \\
\hline PLR & & & 0.622 & 0.430 \\
\hline$<197$ & $183(76.6 \%)$ & 74 (72.5\%) & & \\
\hline$\geq 197$ & $56(23.4 \%)$ & $28(27.5 \%)$ & & \\
\hline BMI, kg/m2 & & & 77.555 & $P<0.01$ \\
\hline$<18$ & 72 (30.1\%) & $14(13.7 \%)$ & & \\
\hline$>27$ & 135 (56.5\%) & 28 (27.5\%) & & \\
\hline $18-24$ & 25 (10.5\%) & 54 (52.9\%) & & \\
\hline $24-27$ & $7(2.9 \%)$ & $6(5.9 \%)$ & & \\
\hline CAI $25, \mathrm{U} / \mathrm{mL}$ & & & 2.104 & 0.147 \\
\hline abnormal & 210 (87.9\%) & $95(93.1 \%)$ & & \\
\hline normal & $29(12.1 \%)$ & 7 (6.9\%) & & \\
\hline Grade & & & $|3.53|$ & 0.001 \\
\hline GI & I3 (5.4\%) & $3(2.9 \%)$ & & \\
\hline $\mathrm{G} 2$ & 206 (86.2\%) & 76 (74.5\%) & & \\
\hline G3 & 20 (8.4\%) & $23(22.5 \%)$ & & \\
\hline Menopause & & & 0.007 & 0.934 \\
\hline no & 207 (86.6\%) & 88 (86.3\%) & & \\
\hline yes & 32 (I3.4\%) & 14 (I3.7\%) & & \\
\hline LVSI & & & 4.150 & 0.042 \\
\hline negative & 236 (98.7\%) & 97 (95.1\%) & & \\
\hline positive & $3(1.3 \%)$ & $5(4.9 \%)$ & & \\
\hline Histology & & & 0.034 & 0.853 \\
\hline Adenocarcinoma & $4(1.7 \%)$ & $2(2.0 \%)$ & & \\
\hline Squamous cell carcinoma & 235 (98.3\%) & $100(98.0 \%)$ & & \\
\hline Surgical margin & & & 0.500 & 0.479 \\
\hline Negative & 233 (97.5\%) & 98 (96.1\%) & & \\
\hline Positive & $6(2.5 \%)$ & $4(3.9 \%)$ & & \\
\hline Parametrial invasion & & & 0.440 & 0.507 \\
\hline negative & $207(86.6 \%)$ & 91 (89.2\%) & & \\
\hline positive & 32 (I3.4\%) & II (I0.8\%) & & \\
\hline
\end{tabular}

(Continued) 
Table I (Continued).

\begin{tabular}{|c|c|c|c|c|}
\hline \multirow[t]{2}{*}{ Variables } & Training Cohort & Validation Cohort & \multirow[t]{2}{*}{$\chi / \mathbf{t}$} & \multirow[t]{2}{*}{$P$-value } \\
\hline & $(n=239)$ & $(n=102)$ & & \\
\hline $\begin{array}{l}\text { Lymph node metastasis } \\
\text { negative } \\
\text { positive }\end{array}$ & $\begin{array}{l}202 \text { (84.5\%) } \\
37(15.5 \%)\end{array}$ & $\begin{array}{l}85(83.3 \%) \\
17(16.7 \%)\end{array}$ & 0.075 & 0.784 \\
\hline $\begin{array}{c}\text { FIGO } \\
\text { lb2 } \\
\text { lla I } \\
\text { lla2 }\end{array}$ & $\begin{array}{l}14(5.9 \%) \\
7(2.9 \%) \\
218(91.2 \%)\end{array}$ & $\begin{array}{l}15(14.7 \%) \\
19(18.6 \%) \\
68(66.7 \%)\end{array}$ & 75.956 & $P<0.01$ \\
\hline $\begin{array}{l}\text { rNACT } \\
\text { yes } \\
\text { no }\end{array}$ & $\begin{array}{l}213(89.1 \%) \\
26(10.9 \%)\end{array}$ & $\begin{array}{l}88(86.3 \%) \\
14(13.7 \%)\end{array}$ & 0.560 & 0.454 \\
\hline
\end{tabular}

Note: Normal CAI 25 values are $<35 \mathrm{kU} / \mathrm{L}$.

Abbreviations: LVSI, lymph vascular space invasion; FIGO, International Federation of Gynecology and Obstetrics.

markers (eg, CA125). The results arising from the univariate logistic analysis are summarized in Table 3. Multivariate analysis showed that NLR $(0.50 ; 95 \% \mathrm{CI}$, 0.32 to 0.79$)$, PLR (3.54, $95 \% \mathrm{CI}, 1.81$ to 6.94$)$, LMR $(0.44,95 \% \mathrm{CI}, 0.21$ to 0.91$)$, platelet count $<100 \times 10^{3} / \mu \mathrm{L}$ (1.99, 95\% CI, 0.92 to 4.35 ), and FIGO stage (IIa1 vs Ib2: $2.89,95 \%$ CI, 0.34 to 4.57 ; Ila2 vs Ib2: $2.43,95 \%$ CI, 0.22 to 3.88 ) were independently associated with rNACT. The results arising from the multivariate logistic analysis are summarized in Table 4. Next, we optimized the model by performing stepwise regression analysis. Decision curve analysis identified that predictive model B (which included FIGO, LMR, NLR, PLR, and platelet count, as they key variables) was the most appropriate model to use when constructing the nomogram (Figures 1B, 2A and B). The nomogram derived from the training cohort was validated using the bootstrap validation method (1000 iterations) with an unadjusted C-index of 0.76 (95\% CI, 0.65 to 0.87). Calibration curves clearly showed that the nomogram produced robust results in the training cohort (Figure 2C). The nomogram also represented an unadjusted C-index of $0.71(95 \% \mathrm{CI}, 0.62$ to 0.78$)$ in the validation cohort. To further test the efficacy of the model, we used clinical impact curves to evaluate stratified risk. The calibration curve showed that the risk of rNACT could be readily distinguished in patients with LACC (Figure 3).

\section{Discussion}

Though concurrent chemoradiation is recommended as the standard treatment for patients with LACC, its complications seriously reduce the patients' quality of life. NACT followed by radical hysterectomy and pelvic lymph node resection is a promising strategy for LACC and exhibits favorable toxicity and comparable or improved survival. ${ }^{7}$ Previous data have shown that rNACT can significantly worsen the survival outcomes of patients with LACC. In the present study, we found that approximately $11 \%$ of patients receiving NACT failed to respond (non-rNACT).

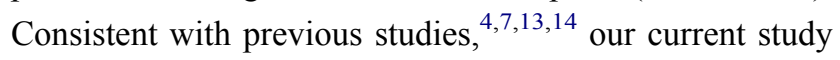
revealed that rNACT was an independent risk factor for prognosis (such as OS and PFS). Our study also demonstrated several factors that were significantly associated with rNACT and could be used as reliable predictive factors, including NLR, PLR, LMR, a reduction in platelet count $\left(<100 \times 10^{3} / \mu \mathrm{L}\right)$, and FIGO stage. Thus, we developed and validated a novel nomogram model to predict the probability of rNACT; we named this the pre-rNACT prediction model (pre-rNACT score). The pre-rNACT score is a visualized scale that is calculated by weighted variables. If a patient requires NACT, then the clinician can take advantage of this pre-rNACT score to assess the risk of rNACT; this may help the clinician to decide on further treatments during the follow-up period. Performance tests showed that the pre-rNACT score exhibited a C-index of 0.76 ( $95 \%$ CI, 0.65 to 0.87 ) in the training cohort and also exhibited robust internal calibration in the validation cohort. In order to verify whether patients could benefit from pre-NACT scoring, we used decision curve analysis and clinical impact curves. We found that the optimal decision cutoff point was achieved when the risk of rNACT threshold was set to 0.64; this 
Table 2 Univariate and Multivariate Cox Regression Analysis of Overall Survival (OS) in Patients with LACC

\begin{tabular}{|c|c|c|c|c|}
\hline \multirow[t]{2}{*}{ Variables } & \multicolumn{2}{|l|}{ Univariate } & \multicolumn{2}{|l|}{ Multivariate } \\
\hline & HR(95\% Cl) & P-value & HR(95\% Cl) & P-value \\
\hline Age & $1.05(1.04-1.06)$ & $<0.01$ & $1.04(1.02-1.05)$ & $<0.01$ \\
\hline Diameter $(<4 \mathrm{~cm}$ vs $\geq 4 \mathrm{~cm})$ & $0.17(0.11-0.27)$ & $<0.01$ & $0.74(0.40-1.36)$ & 0.02 \\
\hline $\operatorname{NLR}(<3.16$ vs $\geq 3.16)$ & $0.64(0.45-0.92)$ & 0.01 & $1.07(0.73-1.58)$ & $<0.01$ \\
\hline $\operatorname{LMR}(<3.6$ vs $\geq 3.6)$ & $0.06(0.05-0.09)$ & $<0.01$ & $0.26(0.14-0.48)$ & $<0.01$ \\
\hline $\operatorname{PLR}(<197$ vs $\geq 197)$ & $2.85(1.67-4.85)$ & $<0.01$ & $1.84(0.82-4.10)$ & 0.04 \\
\hline Menopause(yes vs no) & $5.73(4.14-7.96)$ & 0.68 & $0.99(0.66-1.49)$ & - \\
\hline LVSI(positive vs negative) & $1.69(1.15-2.47)$ & $<0.01$ & $1.64(0.98-2.76)$ & 0.04 \\
\hline Surgical margin (positive vs negative) & & & $\mathrm{I} .33(0.73-2.4 \mathrm{I})$ & - \\
\hline Lymphnode metastasis (positive vs negative) & $0.99(0.63-1.55)$ & 0.97 & $0.78(0.47-1.3 I)$ & - \\
\hline CAI25 (normal vs abnormal) & 6.96(4.97-9.74) & $<0.01$ & $0.87(0.56-1.34)$ & - \\
\hline Parametrial invasion(yes vs no) & $5.74(4.14-7.96)$ & $<0.01$ & $0.72(0.5 \mathrm{I}-\mathrm{I} .23)$ & - \\
\hline Platelet count ( $<300$ vs $\geq 300$ ) & $0.59(0.24-1.79)$ & 0.04 & $0.77(0.25-1.39)$ & 0.03 \\
\hline response to NACT (yes vs no) & $2.74(1.40-5.38)$ & $<0.01$ & $3.09(1.49-6.39)$ & $<0.01$ \\
\hline \multicolumn{5}{|l|}{ BMI } \\
\hline$<18$ & reference & & & \\
\hline$>27$ & $1.97(1.26-3.07)$ & $<0.01$ & $0.74(0.44-1.24)$ & - \\
\hline $18-24$ & $1.63(0.99-2.67)$ & 0.05 & $0.42(0.24-0.73)$ & - \\
\hline $24-27$ & $2.44(1.33-4.48)$ & $<0.01$ & $0.40(0.18-0.90)$ & - \\
\hline \multicolumn{5}{|l|}{ Grade } \\
\hline GI & reference & & & \\
\hline G2 & $0.3 I(0.19-0.5 I)$ & $<0.01$ & $0.92(0.56-1.53)$ & $<0.01$ \\
\hline G3 & $0.2 \mathrm{I}(0.09-0.47)$ & $<0.01$ & $1.79(0.73-4.35)$ & $<0.01$ \\
\hline \multicolumn{5}{|l|}{ FIGO } \\
\hline $\mathrm{lb} 2$ & reference & & & \\
\hline llal & $5.23(1.87-23.90)$ & 0.01 & $5.26(0.61-9.63)$ & $<0.01$ \\
\hline lla2 & $10.64(1.49-16.09)$ & 0.02 & $3.26(0.39-7.7 I)$ & $<0.01$ \\
\hline
\end{tabular}

represented a point at which there was significant discriminative power.

Previous studies have attempted to use preoperative serum hemoglobin level ${ }^{15}$ and pretreatment platelet count $^{16}$ to predict rNACT; however, these earlier studies both used small sample sizes and require further clinical evaluation. Park et al reported that excision repair crosscomplementation facilitated the prediction of responses to cisplatin-based NACT in patients with stage 2B LACC. ${ }^{17}$ However, other factors that have previously been confirmed to be useful for the evaluation of rNACT were not fully adopted in these earlier studies. Of the currently optimized predictive tools, the nomogram score is outstanding with regard to high accuracy and good levels of discrimination. ${ }^{18}$ When considered as a whole, our prerNACT score showed reliable internal calibration between actual assessments and predictions; the rNACT score also performed at a robust level in the validation cohort.
Previous studies have routinely used novel serum biomarkers to create nomograms. In contrast, we created an accurate nomogram by combining the results of routine blood tests, imaging indicators, and serological tumor indicators. These variables are the most common clinical indicators and are therefore very easy to acquire. Other indicators can therefore be avoided, thus reducing the financial burden incurred by patients. Interestingly, most of these indicators are associated with inflammation. Existing studies have confirmed that some systemic inflammatory indicators can comprehensively reflect the inflammatory and immune status of patients with a variety of malignant tumors. ${ }^{19,20}$ The tumor immunoediting theory highlights the fact that different stages of the inflammatory response can contribute to the generation

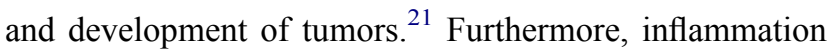
can promote the migration of neutrophils around tumor cells, release reactive oxygen species that induce DNA 
A

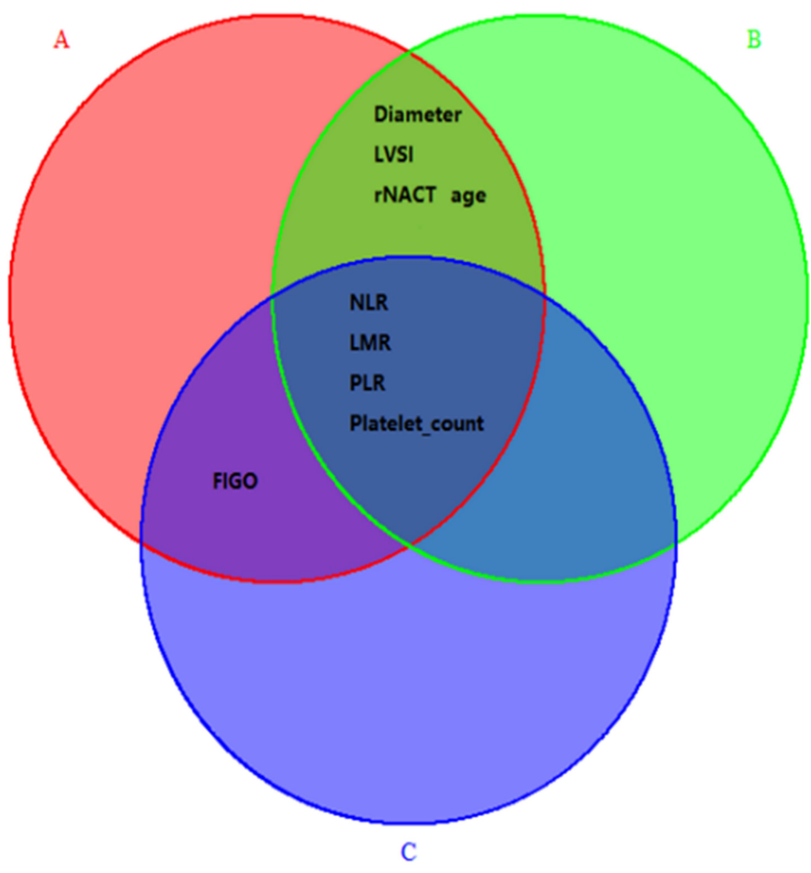

B

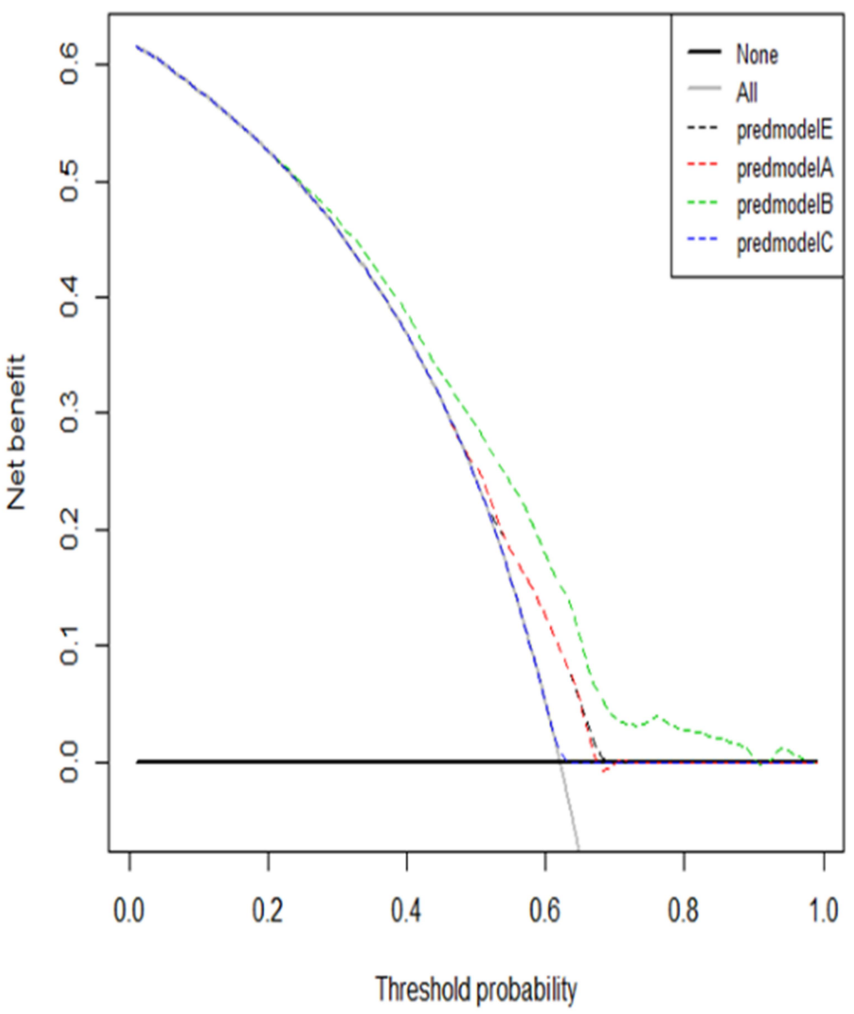

Figure I A Venn diagram showing risk and diagnosis factors (A) and decision curve analysis (B). (A) All prognostic factors for OS are included in circle A and all prognostic factors for PFS are included in circle B. The risk factors for rNACT are shown in circle C. The intersection of the Venn diagram shows the common influential factors. (B) Decision curve for the prediction of $r$ NACT. Decision curve analysis identified potential factors that can exert clinical influence based on stepwise regression analysis and the net benefit of using rNACT score to stratify patients. Four models were built before the final nomogram was constructed: predmodelA (FIGO, LMR, NLR, age, and PLR), predmodelB (FIGO, LMR, NLR, platelet count, and PLR), predmodelC (FIGO, age), and predmodelE (LMR, NLR, and PLR).

damage, and create a suitable microenvironment for tumor development. $^{22}$ Neutrophils promote the production of vascular endothelial growth factor (VEGF) from tumors and accelerate the process of distant metastasis. Lymphocytes can differentiate into tumor-infiltrating lymphocytes within the tumor microenvironment and participate in the adaptive immune response. ${ }^{23,24}$ Research has also shown that monocytes can differentiate into macrophages in the tumor microenvironment, promote angiogenesis, and accelerate tumor migration and metastasis. ${ }^{25,26}$ The activation of platelets can promote tumor angiogenesis, degrade the extracellular matrix, induce the release of adhesion molecules and growth factors, and promote tumor growth and metastasis. ${ }^{27}$ The predictive value of peripheral blood inflammatory markers for the prognosis of malignant tumors requires further investigation.

Previous studies have shown that peripheral blood inflammatory markers are associated with the prognosis of small cell lung cancer, ${ }^{28}$ bladder cancer, ${ }^{29}$ hepatocellular carcinoma, ${ }^{30}$ gastrointestinal cancers, ${ }^{31}$ and other forms of malignancy. ${ }^{32-34}$ Our present study also demonstrated that NLR, PLR, and LMR, were all significantly associated with rNACT. In addition, we found that a reduction in platelet count was associated with an increased risk of non-rNACT in patients with LACC. When applied clinically, this nomogram might serve as a tool to select patients for preoperative NACT or provide clinicians with an opportunity to select alternative treatment options. It is important to remember that rNACT is not the only factor to consider when deciding on therapeutic procedures for LACC. Consequently, it is very important to choose an appropriate initial treatment plan for patients with LACC. Compared with a single indicator, the combination of peripheral blood NLR, PLR, and LMR clearly exhibited a higher predictive value for sensitivity with regards to neoadjuvant chemotherapy. Furthermore, 
Table 3 Univariate and Multivariate Cox Regression Analysis of Progression-Free Survival (PFS) in Patients with LACC

\begin{tabular}{|c|c|c|c|c|}
\hline \multirow[t]{2}{*}{ Variables } & \multicolumn{2}{|l|}{ Univariate } & \multicolumn{2}{|l|}{ Multivariate } \\
\hline & HR(95\% Cl) & P-value & HR(95\% Cl) & P-value \\
\hline Age & $1.06(1.04-1.07)$ & $<0.01$ & $1.04(1.02-1.07)$ & $<0.01$ \\
\hline Diameter $(<4 \mathrm{~cm}$ vs $\geq 4 \mathrm{~cm})$ & $0.17(0.09-0.33)$ & $<0.01$ & $0.89(0.34-2.34)$ & $<0.01$ \\
\hline $\operatorname{NLR}(<3.16$ vs $\geq 3.16)$ & $0.5 I(0.28-0.93)$ & 0.03 & $1.37(0.69-2.72)$ & $<0.01$ \\
\hline $\operatorname{LMR}(<3.6$ vs $\geq 3.6)$ & $0.04(0.02-0.08)$ & $<0.01$ & $0.13(0.04-0.43)$ & $<0.01$ \\
\hline $\operatorname{PLR}(<197$ vs $\geq 197)$ & $1.47(0.72-3.02)$ & 0.02 & $0.37(0.16-0.88)$ & $<0.01$ \\
\hline Menopause(yes vs no) & $6.27(3.71-10.59)$ & 0.61 & $0.76(0.39-1.50)$ & - \\
\hline LVSI(positive vs negative) & $2.37(1.35-4.18)$ & $<0.01$ & $0.17(0.65-4.47)$ & 0.02 \\
\hline Surgical margin (positive vs negative) & $0.92(0.39-2.12)$ & 0.84 & $0.42(0.16-1.13)$ & - \\
\hline Lymphnode metastasis (positive vs negative) & $0.78(0.35-1.70)$ & 0.52 & $0.71(0.27-1.63)$ & - \\
\hline CAI25 (normal vs abnormal) & $8.42(4.83-14.68)$ & $<0.01$ & $0.74(0.33-1.66)$ & - \\
\hline Parametrial invasion(yes vs no) & $6.27(3.71-10.59)$ & $<0.01$ & $0.56(0.4 I-1.68)$ & - \\
\hline Platelet count ( $<300$ vs $\geq 300$ ) & $0.67(0.31-1.86)$ & 0.04 & $0.27(0.21-1.45)$ & 0.03 \\
\hline response to NACT (yes vs no) & $2.46(0.89-6.81)$ & 0.01 & $1.49(0.50-4.46)$ & $<0.01$ \\
\hline \multicolumn{5}{|l|}{ BMI } \\
\hline$<18$ & reference & & & \\
\hline$>27$ & $0.58(0.24-1.39)$ & 0.21 & $0.25(0.09-0.65)$ & - \\
\hline $18-24$ & $3.22(1.59-6.52)$ & 0.08 & $1.35(0.59-3.05)$ & - \\
\hline $24-27$ & $5.87(2.65-12.98)$ & 0.12 & $2.28(0.68-7.64)$ & - \\
\hline \multicolumn{5}{|l|}{ Grade } \\
\hline GI & reference & & & \\
\hline G2 & $2.24(0.31-16.36)$ & 0.42 & $1.94(1.25-7.31)$ & - \\
\hline G3 & $1.41(0.19-15.38)$ & 0.99 & $1.59(0.37-5.13)$ & - \\
\hline \multicolumn{5}{|l|}{ FIGO } \\
\hline $\mathrm{lb} 2$ & reference & & & \\
\hline llal & $2.76(1.69-13.45)$ & 0.23 & $3.11(0.26-17.58)$ & - \\
\hline lla2 & $3.64(1.53-14.79)$ & 0.27 & $3.51(0.16-\mid 6.71)$ & - \\
\hline
\end{tabular}

Table 4 Univariate and Multivariate Logistic Regression Analysis for Risk Factors Associated with rNACT in Patients with LACC

\begin{tabular}{|c|c|c|c|c|}
\hline \multirow[t]{2}{*}{ Variables } & \multicolumn{2}{|l|}{ Univariate } & \multicolumn{2}{|l|}{ Multivariate } \\
\hline & OR(95\% Cl) & P-value & OR(95\% Cl) & P-value \\
\hline Age* & $1.46(0.99-2.14)$ & 0.05 & $1.89(1.03-3.47)$ & 0.14 \\
\hline $\operatorname{NLR}(<3.16$ vs $\geq 3.16)$ & $0.5 \mathrm{I}(0.33-0.78)$ & $<0.01$ & $0.50(0.32-0.79)$ & $<0.01$ \\
\hline $\operatorname{LMR}(<3.6$ vs $\geq 3.6)$ & $0.95(0.61-1.49)$ & 0.04 & $0.44(0.21-0.91)$ & 0.02 \\
\hline $\operatorname{PLR}(<197$ vs $\geq 197)$ & $2.39(1.36-4.19)$ & $<0.01$ & $3.54(1.81-6.94)$ & $<0.01$ \\
\hline Plate count ( $<300$ vs $\geq 300$ ) & $1.69(0.94-3.05)$ & 0.07 & $1.99(0.92-4.35)$ & 0.03 \\
\hline \multicolumn{5}{|l|}{ FIGO } \\
\hline lb2 & reference & & & \\
\hline llal & $2.24(1.07-3.52)$ & 0.04 & $2.89(0.34-4.57)$ & 0.04 \\
\hline Ila2 & $2.52(0.28-3.95)$ & 0.01 & $2.43(0.22-3.88)$ & 0.03 \\
\hline
\end{tabular}

Note: *Continuous variable.

this is a convenient, simple, economical, and reproducible test to perform. Our nomogram provides us with a method of evaluating the biological characteristics and prognoses of patients with LACC more accurately.
Nevertheless, the present study has several limitations that need to be considered. First, the pre-rNACT nomogram score scale was only applied to patients with stage Ib2 to IIa2 (a narrow range); further research is required to 
A

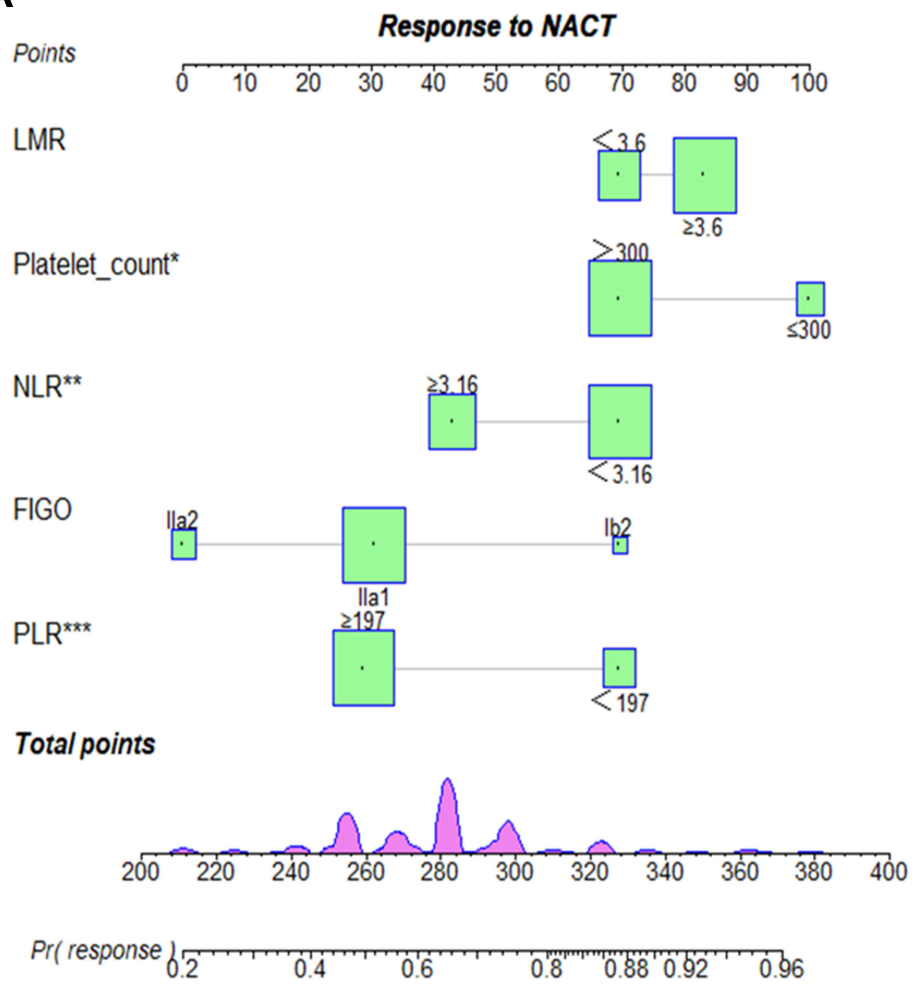

B

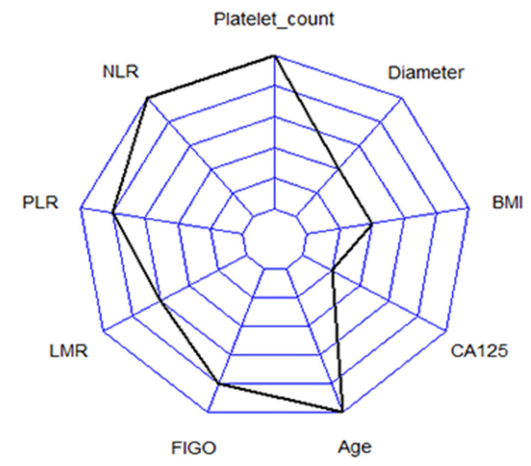

C

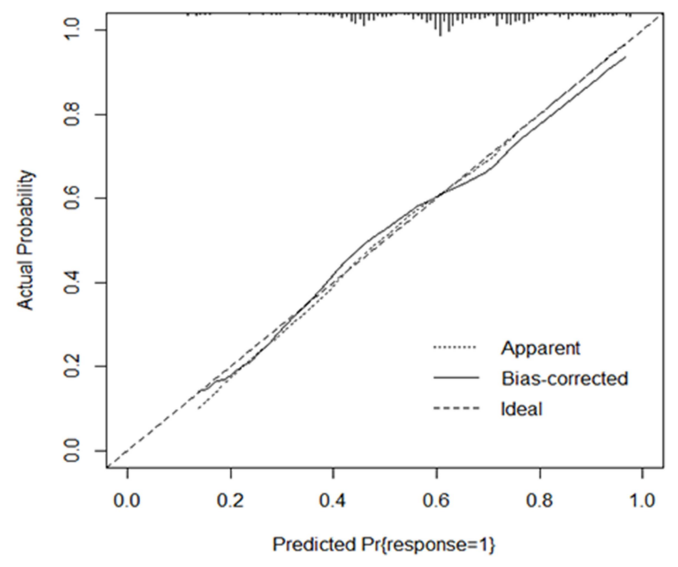

Figure 2 Nomogram to estimate the risk of rNACT. (A) A nomogram for predicting the risk of rNACT showing the proportion (\%) of parameters included in the score scale. To use the rNACT nomogram score, it is important to identify the point of each variable on the corresponding axis; the total number of points can then be summated from all variables. (B) Radar plot showing the relative weight of candidate parameters arising from stepwise regression analysis. The largest proportion is accounted for by NLR, PLR, and platelet count. (C) Calibration curves depicting the robust performance of the nomogram in terms of consensus between the predicted risk and actual risk assessment.

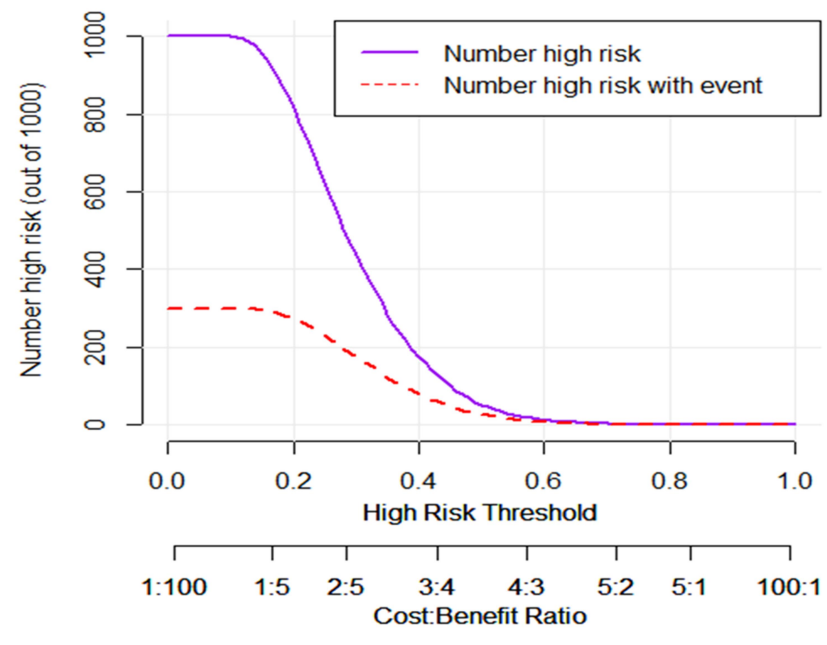

Figure 3 Clinical impact curve for the rNACT nomogram score. The purple line predicts the probability of patients who would show a low/poor response to the NACT. The red line calculated for predmodelB shows how many patients would be at a high risk of non-rNACT. investigate patients who do not meet these criteria (eg, stage Ib2 to Iva; a broader range). Second, this study was based on data from a single institution. Although our validation was robust, it is now necessary to conduct external validation using data from other centers. Third, because the model parameters were based on clinical peripheral blood and imaging results, the application of specific technologies (such as immunological diagnosis biomarkers) might improve the accuracy of our nomogram. Future research should be cautious and validated carefully.

\section{Conclusion}

We developed the pre-rNACT score scale, a novel nomogram-based prediction model featuring peripheral blood and imaging results that can provide an optimized preoperative estimation of the risk of rNACT in patients with LACC. 


\section{Acknowledgments}

The authors gratefully acknowledge all of our participants for sharing their medical records. The authors also wish to thank the staff members at Hubei Cancer Hospital for their assistance with data collection. The authors also thank Charlesworth Author Services for their professional English editing service.

\section{Funding}

This research was supported by the Natural Science Foundation of Hubei Province (No.2019CFB643).

\section{Disclosure}

None of the authors have any conflicts of interest to declare.

\section{References}

1. Cohen PA, Jhingran A, Oaknin A, et al. Cervical cancer. Lancet. 2019;393(10167):169-182. doi:10.1016/S0140-6736(18)32470-X

2. Liu Y, Wu L, Tong R, et al. PD-1/PD-L1 inhibitors in cervical cancer. Front Pharmacol. 2019;10:65. doi:10.3389/fphar.2019.00065

3. Lapresa M, Parma G, Portuesi R, et al. Neoadjuvant chemotherapy in cervical cancer: an update. Expert Rev Anticancer Ther. 2015;15 (10):1171-1181. doi:10.1586/14737140.2015.1079777

4. Li R, Lu S-T, Si J-G, et al. Prognostic value of responsiveness of neoadjuvant chemotherapy before surgery for patients with stage IB (2)/IIA(2) cervical cancer. Gynecol Oncol. 2013;128(3):524-529. doi:10.1016/j.ygyno.2012.11.006

5. Gadducci A, Cosio S. Neoadjuvant chemotherapy in locally advanced cervical cancer: review of the literature and perspectives of clinical research. Anticancer Res. 2020;40(9):4819-4828. doi:10.21873/ anticanres. 14485

6. Moore DH. Neoadjuvant chemotherapy for cervical cancer. Expert Opin Pharmacother. 2003;4(6):859-867. doi:10.1517/14656566.4.6.859

7. Huang Y, Liu L, Cai J, et al. The efficacy and response predictors of platinum-based neoadjuvant chemotherapy in locally advanced cervical cancer. Cancer Manag Res. 2020;12:10469-10477. doi:10.2147/ CMAR.S270258

8. Nishino M. Tumor response assessment for precision cancer therapy: response evaluation criteria in solid tumors and beyond. Am Soc Clin Oncol Educ Book. 2018;38(38):1019-1029. doi:10.1200/EDBK_201441

9. Eisenhauer EA, Therasse P, Bogaerts J, et al. New response evaluation criteria in solid tumours: revised RECIST guideline (version 1.1). Eur J Cancer. 2009;45(2):228-247. doi:10.1016/j.ejca.2008.10.026

10. Steyerberg EW, Vergouwe Y. Towards better clinical prediction models: seven steps for development and an ABCD for validation. Eur Heart J. 2014;35(29):1925-1931. doi:10.1093/eurheartj/ehu207

11. Vickers AJ, Cronin AM, Elkin EB, et al. Extensions to decision curve analysis, a novel method for evaluating diagnostic tests, prediction models and molecular markers. BMC Med Inform Decis Mak. 2008;8 (1):53. doi:10.1186/1472-6947-8-53

12. Vickers AJ, Elkin EB. Decision curve analysis: a novel method for evaluating prediction models. Med Decis Making. 2006;26 (6):565-574. doi:10.1177/0272989X06295361

13. Benson R, Pathy S, Kumar L, et al. Locally advanced cervical cancer - neoadjuvant chemotherapy followed by concurrent chemoradiation and targeted therapy as maintenance: a Phase II study. $J$ Cancer Res Ther. 2019;15(6):1359-1364. doi:10.4103/jcrt. JCRT_39_18
14. Ye Q, Yuan H-X, Chen H-L. Responsiveness of neoadjuvant chemotherapy before surgery predicts favorable prognosis for cervical cancer patients: a meta-analysis. J Cancer Res Clin Oncol. 2013;139 (11):1887-1898. doi:10.1007/s00432-013-1509-y

15. Fuso L, Mazzola S, Marocco F, et al. Pretreatment serum hemoglobin level as a predictive factor of response to neoadjuvant chemotherapy in patients with locally advanced squamous cervical carcinoma: a preliminary report. Gynecol Oncol. 2005;99(Suppl 3):S187-91. doi:10.1016/j.ygyno.2005.07.079

16. Gadducci A, Cosio S, Zola P, et al. Pretreatment platelet and hemoglobin levels are neither predictive nor prognostic variables for patients with locally advanced cervical cancer treated with neoadjuvant chemotherapy and radical hysterectomy: a retrospective Italian study. Int J Gynecol Cancer. 2010;20(8):1399-1404. doi:10.1111/ IGC.0b013e3181f1574e

17. Park J-S, Jeon EK, Chun SH, et al. ERCC1 (excision repair cross-complementation group 1) expression as a predictor for response of neoadjuvant chemotherapy for FIGO stage 2B uterine cervix cancer. Gynecol Oncol. 2011;120(2):275-279. doi:10.1016/j. ygyno.2010.10.034

18. Shariat SF, Capitanio U, Jeldres C, et al. Can nomograms be superior to other prediction tools? BJU Int. 2009;103(4):492-5; discussion 495-7. doi:10.1111/j.1464-410X.2008.08073.x

19. Kumar R, Geuna E, Michalarea V, et al. The neutrophil-lymphocyte ratio and its utilisation for the management of cancer patients in early clinical trials. Br J Cancer. 2015;112(7):1157-1165. doi:10.1038/ bjc. 2015.67

20. Roxburgh CS, McMillan DC. Role of systemic inflammatory response in predicting survival in patients with primary operable cancer. Future Oncol. 2010;6(1):149-163. doi:10.2217/fon.09.136

21. Bui JD, Schreiber RD. Cancer immunosurveillance, immunoediting and inflammation: independent or interdependent processes? Curr Opin Immunol. 2007;19(2):203-208. doi:10.1016/j.coi.2007.02.001

22. Coussens LM, Werb Z. Inflammation and cancer. Nature. 2002;420 (6917):860-867. doi:10.1038/nature01322

23. Kusumanto YH, Dam WA, Hospers GAP, et al. Platelets and granulocytes, in particular the neutrophils, form important compartments for circulating vascular endothelial growth factor. Angiogenesis. 2003;6(4):283-287. doi:10.1023/B:AGEN.0000029415.62384.ba

24. Colotta F, Allavena P, Sica A, et al. Cancer-related inflammation, the seventh hallmark of cancer: links to genetic instability. Carcinogenesis. 2009;30(7):1073-1081. doi:10.1093/carcin/bgp127

25. Lin EY, Li J-F, Gnatovskiy L, et al. Macrophages regulate the angiogenic switch in a mouse model of breast cancer. Cancer Res. 2006;66(23):11238-11246. doi:10.1158/0008-5472.CAN-06-1278

26. Lee Y, Kim SH, Han J-Y, et al. Early neutrophil-to-lymphocyte ratio reduction as a surrogate marker of prognosis in never smokers with advanced lung adenocarcinoma receiving gefitinib or standard chemotherapy as first-line therapy. J Cancer Res Clin Oncol. 2012;138 (12):2009-2016. doi:10.1007/s00432-012-1281-4

27. Egan K, Crowley D, Smyth P, et al. Platelet adhesion and degranulation induce pro-survival and pro-angiogenic signalling in ovarian cancer cells. PLoS One. 2011;6(10):e26125. doi:10.1371/journal.pone.0026125

28. Liu F, Zhou S, Tan L, et al. A retrospective cohort study on pretreated neutrophil-to-lymphocyte ratio and prognosis of small cell lung cancer: evidence of effect modification by chemotherapy regimen. Cancer Manag Res. 2020;12:10341-10352. doi:10.2147/CMAR.S263863

29. Lee SM, Russell A, Hellawell G. Predictive value of pretreatment inflammation-based prognostic scores (neutrophil-to-lymphocyte ratio, platelet-to-lymphocyte ratio, and lymphocyte-to-monocyte ratio) for invasive bladder carcinoma. Korean J Urol. 2015;56 (11):749-755. doi:10.4111/kju.2015.56.11.749

30. Yoneoka G, Bozhilov K, Wong LL. Prognostic ability of inflammation-based markers in radioembolization for hepatocellular carcinoma. Hepatoma Res. 2020;6:6. doi:10.20517/2394-5079. 2020.57 
31. Bowen RC, Little NAB, Harmer JR, et al. Neutrophil-to-lymphocyte ratio as prognostic indicator in gastrointestinal cancers: a systematic review and meta-analysis. Oncotarget. 2017;8(19):32171-32189. doi:10.18632/oncotarget.16291

32. Yang C, Wen H-B, Zhao Y-H, et al. Systemic inflammatory indicators as prognosticators in glioblastoma patients: a comprehensive meta-analysis. Front Neurol. 2020;11:580101. doi:10.3389/fneur. 2020.580101
33. Zhu Y, Zhou S, Liu Y, et al. Prognostic value of systemic inflammatory markers in ovarian Cancer: a PRISMA-compliant meta-analysis and systematic review. BMC Cancer. 2018;18(1):443. doi:10.1186/ s12885-018-4318-5

34. Ethier J-L, Desautels D, Templeton A, et al. Prognostic role of neutrophil-to-lymphocyte ratio in breast cancer: a systematic review and meta-analysis. Breast Cancer Res. 2017;19(1):2. doi:10.1186/ s13058-016-0794-1

\section{Publish your work in this journal}

Cancer Management and Research is an international, peer-reviewed open access journal focusing on cancer research and the optimal use of preventative and integrated treatment interventions to achieve improved outcomes, enhanced survival and quality of life for the cancer patient.
The manuscript management system is completely online and includes a very quick and fair peer-review system, which is all easy to use. Visit http://www.dovepress.com/testimonials.php to read real quotes from published authors. 